\title{
A GENERALIZATION OF PRIMARY IDEALS AND STRONGLY PRIME SUBMODULES
}

\author{
AFROOZEH JAFARI, MOHAMMAD BAZIAR, AND SAEED SAFAEEYAN
}

\begin{abstract}
We present *-primary submodules, a generalization of the concept of primary submodules of an $R$-module. We show that every primary submodule of a Noetherian $R$-module is $*$-primary. Among other things, we show that over a commutative domain $R$, every torsion free $R$-module is $*$-primary. Furthermore, we show that in a cyclic $R$-module, primary and $*$-primary coincide. Moreover, we give a characterization of $*$-primary submodules for some finitely generated free $R$-modules.
\end{abstract}

\section{INTRODUCTION}

Throughout this paper, all rings are commutative with identity and all modules are unital. A proper ideal $I$ of a ring $R$ is called a prime (resp. primary) ideal if whenever $a b \in I$, where $a, b \in R$, then either $a \in I$ or $b \in I$ (resp. $a \in I$ or $b^{n} \in I$ for some positive integer $n$ ). The notions of prime and primary ideals have been generalized to modules by various authors ([3], [4] and [6]). Let $R$ be a ring, $M$ an $R$-module and $N$ a submodule of $M$. The annihilator of the $R$-module $\frac{M}{N}$ is denoted by $(N: M)$. A proper submodule $N$ of $M$ is called prime (resp. primary) if whenever $r m \in N$, where $r \in R, m \in M$, then either $m \in N$ or $r \in(N: M)$ (resp. $m \in N$ or $r^{n} \in(N: M)$ for some positive integer $n$ ). For more details about prime and primary submodules one can see [2, [5], 7], [11] and [12. For a proper submodule $N$ of an $R$-module $M$ and $a \in R$, set $\left(N:_{M} a\right)=\{m \in M \mid a m \in N\}$. It is easy to show that $\left(N:_{M} a\right)$ is a submodule of $M$. Following [1, a proper submodule $N$ of $M$ is said to be classical primary, if $a b m \in N$, where $a, b \in R$, $m \in M$, implies that $a m \in N$ or $b^{n} m \in N$ for some $n \in \mathbb{N}$.

Strongly prime submodules have been introduced and studied in [8] and $[9]$. According to [8], a proper submodule $N$ of an $R$-module $M$ is said to be strongly prime provided that $(R x+N: M) y \subseteq N$, for $x, y \in M$, implies that either $x \in N$ or $y \in N$.

In this paper we introduce and investigate $*$-primary submodules, which are a generalization of primary ideals and strongly prime submodules.

2020 Mathematics Subject Classification. 13C13, 13E05, 13E15.

Key words and phrases. Primary ideal; Primary submodule; *-prime submodule; *-primary submodule. 
Definition 1.1. A proper submodule $N$ of an $R$-module $M$ is called *-primary (resp. *-prime) if $(R x+N: M) y \subseteq N$ for $x, y \in M$ implies that either $y \in N$ or $(R x+N: M)^{k} \subseteq(N: M)$ for some $k \in \mathbb{N}$ (resp. $y \in N$ or $(R x+N: M) \subseteq(N$ : $M))$. If the zero submodule of $M$ is *-primary, $M$ is called *-primary. Moreover the $R$-module $M$ is called fully $*$-primary provided that every proper submodule of $M$ is *-primary.

We have the following diagram which shows the relationship of strongly prime, prime, primary, $*$-prime and $*$-primary submodules.

$$
\begin{aligned}
& \text { strongly prime } \Longrightarrow \text { prime } \Longrightarrow \text { primary } \\
& \Downarrow \\
& \text { *-prime } \Longrightarrow * \text {-primary }
\end{aligned}
$$

We give an example which shows that the classical primary submodule and *-primary submodules are different. Considering $\mathbb{Q}$ as a $\mathbb{Z}$-module, we observe that $\mathbb{Z}$ is a $*$-primary submodule which is neither a classical primary nor a primary submodule. Moreover, for some prime number $p$, the submodule $p \mathbb{Z} \oplus 0$ is a classical primary submodule of $\mathbb{Z} \oplus \mathbb{Z}$ which is not a $*$-primary submodule (see Example 1.2 ).

In the next example we show that the class of $*$-primary submodules is quite different from the class of strongly prime submodules and the class of primary submodules.

Example 1.2. (a) Consider $\mathbb{Q}$ as a $\mathbb{Z}$-module. For every proper submodule $N$ of $\mathbb{Q}$, we have $(N: \mathbb{Q})=0$. So all proper submodules of $\mathbb{Q}$ are $*$-prime and hence *-primary (one can easily extend this fact to divisible modules over a domain). We know that $\mathbb{Q}$ has no nonzero prime (primary) submodule; therefore it has no nonzero strongly prime submodule.

(b) *-primary submodules of the $\mathbb{Z}$-module $\mathbb{Z}_{n}$ are exactly the primary ideals of the ring $\mathbb{Z}_{n}$.

(c) If $(N: M)=m$ is a maximal ideal of $R$, then $N$ is a $*$-primary submodule. In particular, if $N$ is a maximal submodule of $M$ then $N$ is a *-primary submodule. Moreover, if $M$ is a finitely generated module in which for every proper submodule $N$ of $M,(0: M)=(N: M)$, then $N$ is a $*$-primary submodule.

(d) In the $\mathbb{Z}$-module $\mathbb{Z} \oplus \mathbb{Z}$, submodules of the form $0 \oplus m \mathbb{Z}, m \mathbb{Z} \oplus 0$ and $p \mathbb{Z} \oplus q \mathbb{Z}$ are not $*$-primary, where $p, q$ are distinct prime numbers and $m \in \mathbb{Z}$. $((\mathbb{Z}(1, q+1)+p \mathbb{Z} \oplus q \mathbb{Z}: \mathbb{Z} \oplus \mathbb{Z})(1,0) \subseteq p \mathbb{Z} \oplus q \mathbb{Z},(1,0) \notin p \mathbb{Z} \oplus q \mathbb{Z}$ and no power of $(\mathbb{Z}(1, q+1)+p \mathbb{Z} \oplus q \mathbb{Z}: \mathbb{Z} \oplus \mathbb{Z})$ is contained in $p q \mathbb{Z})$.

In Section 2, we show that in a Noetherian $R$-module, every primary submodule is $*$-primary (Proposition 2.8); we also show that torsion free $R$-modules over commutative domains are $*$-primary (Proposition 2.11). In Section 3, we show that for any cyclic $R$-module $M$ and submodule $N$ of $M$, we have that $N$ is strongly prime if and only if $N$ is a $*$-primary submodule with $\frac{R}{(N: M)}$ a reduced ring (Proposition 3.1. Moreover, we show that over a cyclic $R$-module the $*$-primary submodules are precisely primary submodules (Theorem 3.2 . Finally, we investigate $*$-primary submodules of a free $R$-module $M$ of rank 2 (Proposition 3.7 and Theorem 3.9). 


\section{2. *-PRIMARY SUBMODULES}

In this section we obtain necessary and sufficient conditions under which a submodule $N$ of $M$ is $*$-primary.

For a submodule $N$ of an $R$-module $M$ set

$$
Z_{N}(M)=\{y \in M \mid(R x+N: M) y \subseteq N \text { for some } x \in M \backslash N\}
$$

Proposition 2.1. Let $M$ be an $R$-module and $N$ a proper submodule of $M$. The following are equivalent:

(1) $N$ is a $*$-primary submodule of $M$.

(2) For every submodule $L$ of $M$ and $x \in M$, if $(R x+N: M) L \subseteq N$ then $L \subseteq N$ or $(R x+N: M)^{n} \subseteq(N: M)$ for some $n \in \mathbb{N}$.

(3) For each $y \in Z_{N}(M) \backslash N$ and $x \in M \backslash N$ such that $(R x+N: M) y \subseteq N$, there exists an $n \in \mathbb{N}$ such that $(R x+N: M)^{n}(M) \subseteq N$.

(4) For each $x \in M$, either $\frac{(R x+N: M)}{(N: M)}$ is a nilpotent ideal or $(N:(R x+N$ : $M))=N$.

(5) $\frac{M}{N}$ is a *-primary $R$-module.

Proof. $(1 \Rightarrow 2)$ Let $(R x+N: M) L \subseteq N$ and $L \nsubseteq N$. Then there exists $l \in L \backslash N$ such that $(R x+N: M) l \subseteq N$. Now by (1), we have $(R x+N: M)^{n} \subseteq(N: M)$ for some $n \in \mathbb{N}$.

$(2 \Rightarrow 3)$ Assume that $y \in Z_{N}(M) \backslash N, x \in M \backslash N$ and $(R x+N: M) y \subseteq N$. Set $L=R y$. By (2) there exists a positive integer $n \in \mathbb{N}$ such that $(R x+N: M)^{n} \subseteq$ $(N: M)$ or, equivalently, $(R x+N: M)^{n} M \subseteq N$.

$(3 \Rightarrow 4)$ For $x \in N$ it is clear that $\frac{(R x+N: M)}{(N: M)}$ is a nilpotent ideal. Assume that $x \in M \backslash N$ and $\left(N:_{M}(R x+N: M)\right) \nsubseteq N$. Then there exists $y \in\left(N:_{M}(R x+N\right.$ : $M)) \backslash N$. Therefore $(R x+N: M) y \subseteq N$, and hence $y \in Z_{N}(M) \backslash N$. By (3), $(R x+N: M)^{n} \subseteq(N: M)$ for some $n \in \mathbb{N}$, which implies that $\frac{(R x+N: M)}{(N: M)}$ is a nilpotent ideal.

$(4 \Rightarrow 1)$ Suppose that $y, x \in M$ are such that $(R x+N: M) y \subseteq N$ and $y \notin N$. By hypothesis, $\frac{(R x+N: M)}{(N: M)}$ is a nilpotent ideal, and hence for some positive integer $n$, $(R x+N: M)^{n} \subseteq(N: M)$.

$(1 \Rightarrow 5)$ Let $\left(\frac{R x+N}{N}: \frac{M}{N}\right)(y+N)=0$. Since $\frac{\frac{M}{N}}{\frac{R x+N}{N}} \cong \frac{M}{R x+N},(R x+N$ : $M)(y+N)=0$. Then $(R x+N: M) y \subseteq N$. By (1), we get $y \in N$ or $(R x+N$ : $M)^{n} \subseteq(N: M)$ for some $n \in \mathbb{N}$. So $y+N=0$ or $\left(\frac{R x+N}{N}: \frac{M}{N}\right)^{n} \subseteq\left(0: \frac{M}{N}\right)$.

$(5 \Rightarrow 1)$ Let $(R x+N: M) y \subseteq N$. Then $\left(\frac{R x+N}{N}: \frac{M}{N}\right)(y+N)=0$. By (5) we have $y+N=N$ or $\left(\frac{R x+N}{N}: \frac{M}{N}\right)^{n} \subseteq\left(0: \frac{M}{N}\right)$. Hence $y \in N$ or $(R x+N: M)^{n} \subseteq$ $(N: M)$. 
Proposition 2.2. Let $M$ be an $R$-module and $N$ a submodule of $M$ with $Z_{N}(M) \neq$ M. The following statements hold:

(1) If $(N: M)$ is a primary ideal of a Noetherian ring $R$, then $N$ is a *-primary submodule.

(2) $Z_{N}(M)=N$ if and only if $(N: M)$ is a semiprime ideal of $R$ and $N$ is a *-primary submodule.

Proof. (1) Let $(R x+N: M) y \subseteq N, y \notin N$ and $(R x+N: M)^{n} \nsubseteq(N: M)$ for every $n \in \mathbb{N}$. We have $(R x+N: M)(R y+N: M) M \subseteq(R x+N: M)(R y+N) \subseteq N$. Then $(R x+N: M)(R y+N: M) \subseteq(N: M)$. Since $(N: M)$ is a primary ideal of $R$, we have $(R y+N: M) \subseteq(N: M)$. So $(R y+N: M) M \subseteq N$ and this is a contradiction.

(2) Let $Z_{N}(M)=N$ and $(R x+N: M) y \subseteq N$, where $x, y \in M$. If $x \in N$, we are done. Otherwise, $y \in Z_{N}(M)=N$. Consequently, suppose that $Z_{N}(M) \neq N$. There exists $y \in Z_{N}(M)$ such that $y \notin N$. By definition of $Z_{N}(M)$ there exists $x \in M \backslash N$ such that $(R x+N: M) y \subseteq N$. Then $(R x+N: M)^{n} \subseteq(N: M)$ and semiprimeness of $(N: M)$ implies that $(R x+N: M) M \subseteq N$. Therefore $Z_{N}(M)=M$ and this is a contradiction.

Proposition 2.3. Let $M$ be an $R$-module and $m$ be a maximal ideal of $R$. Then $m^{n} M$ is a *-primary submodule of $M\left(m^{n} M \neq M, n \in \mathbb{N}\right)$.

Proof. Let $\left(R x+m^{n} M: M\right) y \subseteq m^{n} M$. If $\left(R x+m^{n} M: M\right) \subseteq m$, then $\left(R x+m^{n} M: M\right)^{n} M \subseteq m^{n} M$. So $\left(R x+m^{n} M: M\right)^{n} \subseteq\left(m^{n} M: M\right)$. If $(R x+$ $\left.m^{n} M: M\right) \nsubseteq m$, then there exists $a \in\left(R x+m^{n} M: M\right)$ such that $a \notin m$. So $a r+b=1, b \in m$. Hence $1=1^{n}=(a r+b)^{n}=b^{n}+s a$ for some $s \in R$. Thus $y=b^{n} y+s a y$, and therefore $y \in m^{n} M$.

In the next proposition we will consider the basic properties of $*$-primary submodules under module homomorphisms.

Proposition 2.4. Let $M$ and $M^{\prime}$ be $R$-modules, $K \subseteq N \subseteq M$ and $f: M \rightarrow M^{\prime}$ an epimorphism. The following statements hold:

(1) If $N^{\prime}$ is a *-primary submodule of $M^{\prime}$, then $f^{-1}\left(N^{\prime}\right)$ is a *-primary submodule of $M .\left(f(M) \nsubseteq N^{\prime}\right)$.

(2) If $N$ is a *-primary submodule of $M$ with ker $f \subseteq N$, then $f(N)$ is a *-primary submodule of $M^{\prime}$.

(3) $N$ is a *-primary submodule of $M$ if and only if $\frac{N}{K}$ is a *-primary submodule of $\frac{M}{K}$.

Proof. (1) Let $\left(R x+f^{-1}\left(N^{\prime}\right): M\right) y \subseteq f^{-1}\left(N^{\prime}\right)$, where $x, y \in M$. We claim that $\left(f(R x)+N^{\prime}: M^{\prime}\right) f(y) \subseteq N^{\prime}$. Let $r \in\left(f(R x)+N^{\prime}: M^{\prime}\right)$. Then $f(r M) \subseteq$ $f(R x)+N^{\prime}$. So for every $m_{0} \in M$ there exists $r_{0} \in R$ such that $f\left(r m_{0}-r_{0} x\right) \in N^{\prime}$. Hence $r m_{0} \in f^{-1}\left(N^{\prime}\right)+R x$. Therefore $r M \subseteq f^{-1}\left(N^{\prime}\right)+R x$. So $r y \in f^{-1}\left(N^{\prime}\right)$, $r f(y) \in N^{\prime}$, and hence $(f(R x)+N: M) y \subseteq N$. Since $N^{\prime}$ is a $*$-primary submodule of $M^{\prime}$, we have that $f(y) \in N^{\prime}$ or $\left(f(R x)+N^{\prime}: M^{\prime}\right)^{n} \subseteq\left(N^{\prime}: M^{\prime}\right)$ for some $n \in \mathbb{N}$. Hence $y \in f^{-1}\left(N^{\prime}\right)$ or $\left(R x+f^{-1}\left(N^{\prime}\right): M\right)^{n} \subseteq\left(f^{-1}\left(N^{\prime}\right): M\right)$. 
(2) Let $\left(R m_{1}^{\prime}+f(N): M^{\prime}\right) m_{2}^{\prime} \subseteq f(N)$, where $m_{1}^{\prime}, m_{2}^{\prime} \in M^{\prime}$. Then there exist $m_{1}, m_{2} \in M$ such that $m_{1}^{\prime}=f\left(m_{1}\right), m_{2}^{\prime}=f\left(m_{2}\right)$. We claim that $\left(R m_{1}+\right.$ $N: M) m_{2} \subseteq N$. Let $r \in\left(R m_{1}+N: M\right)$; then $r f(M) \subseteq R f\left(m_{1}\right)+f(N)$. By assumption, $r f\left(m_{2}\right) \in f(N)$. So $f\left(r m_{2}-n_{0}\right)=0$ for some $n_{0} \in \mathbb{N}$ and hence $r m_{2} \in N$. Since $N$ is a $*$-primary submodule, we have that $m_{2} \in N$ or $\left(R m_{1}+N: M\right)^{n} \subseteq(N: M)$ for some $n \in \mathbb{N}$. So $m_{2}^{\prime}=f\left(m_{2}\right) \in f(N)$ or $\left(R f\left(m_{1}\right)+f(N): M^{\prime}\right)^{n} \subseteq(f(N): f(M))$ for some $n \in \mathbb{N}$.

(3) It is clear by (1) and (2).

Corollary 2.5. Let $M, N, M_{1}$ and $M_{2}$ be $R$-modules.

(1) If $f: M \rightarrow N$ is an epimorphism and $\operatorname{Rad}(M)$ a *-primary submodule, such that $\operatorname{ker} f \subseteq \operatorname{Rad}(M)$ and $\frac{M}{\operatorname{Rad}(M)}$ is a semisimple $R$-module, then $\operatorname{Rad}(N)$ is a $*$-primary submodule of $N$.

(2) Let $N_{1}, N_{2}$ be submodules of $M_{1}, M_{2}$. Then $N_{1}, N_{2}$ are $*$-primary if and only if $N_{1} \oplus M_{2}$ and $M_{1} \oplus N_{2}$ are $*$-primary submodules of $M_{1} \oplus M_{2}$.

In Proposition 2.4 the surjectivity of $f$ is necessary. For example, consider the homomorphism $\varphi: \mathbb{Z} \rightarrow \mathbb{Q}$ via $\varphi(x)=x$. The submodule $6 \mathbb{Z}$ is a $*$-primary submodule in $\mathbb{Q}$, but it is not $*$-primary in $\mathbb{Z}$. For the homomorphism $f: \mathbb{Z} \rightarrow \mathbb{Z} \oplus \mathbb{Z}$ via $f(x)=(x, 0)$, we have $2 \mathbb{Z} \supsetneqq \mathbb{Z}$ is a $*$-primary submodule but $f(2 \mathbb{Z})=2 \mathbb{Z} \oplus 0 \supsetneqq$ $\mathbb{Z} \oplus \mathbb{Z}$ is not a $*$-primary submodule. Also the condition $\operatorname{ker} \varphi \subseteq N$ is necessary. For example, for the surjective homomorphism $\varphi: \mathbb{Z} \rightarrow \mathbb{Z}_{6}$, the zero submodule of $\mathbb{Z}$ is $*$-primary but the submodule $\varphi(0)=\overline{0} \supsetneqq \mathbb{Z}_{6}$ is not $*$-primary.

If $N_{1} \supsetneqq M_{1}$ and $N_{2} \supsetneqq M_{2}$ are $*$-primary, then we cannot always say that $N_{1} \oplus N_{2} \supsetneqq M_{1} \oplus M_{2}$ is a $*$-primary submodule. For example, for every prime number $p \in \mathbb{Z}$, the submodule $p \mathbb{Z} \oplus 0 \supsetneqq \mathbb{Z} \oplus \mathbb{Z}$ is not a $*$-primary submodule.

Fact 2.6. Let $M$ be an $R$-module and $N_{1}$ and $N_{2}$ submodules of $M$.

(1) If the intersection of two submodules is a $*$-primary submodule, then not all of them are necessarily $*$-primary; consider for example $M=\mathbb{Z} \oplus \mathbb{Z}$, $N_{1}=0 \oplus 2 \mathbb{Z}, N_{2}=2 \mathbb{Z} \oplus 0$. Also, the intersection of two *-primary submodules is not necessarily $*$-primary; for example, take $\mathbb{Z}_{6}$ as a $\mathbb{Z}$ module. $\langle\overline{2}\rangle,\langle\overline{3}\rangle$ are $*$-primary. But $\langle\overline{2}\rangle \cap\langle\overline{3}\rangle=\langle\overline{0}\rangle$ is not a $*$-primary submodule.

(2) The property of being *-primary in submodules of $M$ is not preserved under isomorphism (for example, $M=\mathbb{Z}, N_{1}=6 \mathbb{Z}, N_{2}=2 \mathbb{Z}$ ).

Proposition 2.7. Let $M$ be an $R$-module such that for submodules $A, B$ and $C$ of $M$ we have $A+(B \cap C)=(A+B) \cap(A+C) ; N$ a *-primary submodule of $M$; and $K$ a submodule of $M$ such that $K \nsubseteq N$ and $(R x+N: K)=(R x+N: M)$ for every $x \in M$. Then $K \cap N$ is a *-primary submodule of $K$.

Proof. Let $(R x+(K \cap N): K) y \subseteq K \cap N$, where $x, y \in K$ and $y \notin K \cap N$. Then $y \notin N$. We claim that $(R x+N: M) y \subseteq N$. Let $r \in(R x+N: M)$; then $r K \subseteq R x+N$. So $r K \subseteq(R x+N) \cap(R x+K)=R x+(K \cap N)$. Hence $r y \in N$. Since $N$ is a $*$-primary submodule, $(R x+N: M)^{n} \subseteq(N: M)$ for some $n \in \mathbb{N}$. So $(R x+N: M)^{n} \subseteq(N \cap K: K)$, and thus $(R x+(K \cap N): K)^{n} \subseteq(N \cap K: K)$. 
In what follows, *-primary submodules of a Noetherian ring are investigated.

Proposition 2.8. Let $R$ be a Noetherian ring and $M$ an $R$-module. The following statements hold:

(1) Primary submodules of $M$ are *-primary.

(2) For a submodule $N$ of $M$, if $\sqrt{(N: M)}$ is a maximal ideal of $R$, then $N$ is a*-primary submodule of $M$.

Proof. (1) Suppose $x, y \in M$ and $(R x+N: M) y \subseteq N$. Since $N$ is a primary submodule, $y \in N$ or $\left\langle x_{1}, x_{2}, \ldots, x_{n}\right\rangle=(R x+N: M) \subseteq \sqrt{(N: M)}$. Then there exist $k_{1}, k_{2}, \ldots, k_{n} \in \mathbb{N}$ such that $x_{1}^{k_{1}} \in(N: M), \ldots, x_{n}^{k_{n}} \in(N: M)$. Then $(x R+N: M)^{k} \subseteq(N: M)$ for some $k \in \mathbb{N}$.

(2) It follows from (1).

In general, the converse of Proposition 2.8(1) is not true (see Example 1.2.

Corollary 2.9. Let $M$ be a Noetherian $R$-module. Then every primary submodule of $M$ is $*$-primary.

For an $R$-module $M$ and a submodule $N$ of $M$, if $(N: M)$ is a maximal ideal then it is clear that $N$ is $*$-primary. The following example shows that in general if $(N: M)$ is a prime ideal of $R$, we cannot expect $N$ to be a $*$-primary submodule of $M$.

Example 2.10. Let $M=\mathbb{Z} \oplus \mathbb{Z}$ as a $\mathbb{Z}$-module and $N=2 \mathbb{Z} \oplus 0$. The ideal $(N: M)=0$ is a prime ideal of $\mathbb{Z}$ but $N$ is not a $*$-primary submodule. For $(\mathbb{Z}(2,2)+2 \mathbb{Z} \oplus 0: \mathbb{Z} \oplus \mathbb{Z})(1,0) \subseteq 2 \mathbb{Z} \oplus 0,(1,0) \notin 2 \mathbb{Z} \oplus 0$ and powers of $(\mathbb{Z}(2,2)+2 \mathbb{Z} \oplus 0$ : $\mathbb{Z} \oplus \mathbb{Z})$ are nonzero.

A nonzero module is a compressible module if it can be embedded in each of its nonzero submodules.

Theorem 2.11. Let $R$ be an integral domain, $M$ an $R$-module and $N$ a proper submodule of $M$. If $\frac{M}{N}$ is a torsion free or compressible module, then $N$ is a $*$-prime (*-primary) submodule. Moreover, $T(M) \supsetneqq M$ is a *-primary submodule.

Proof. Let $L$ be a submodule of $M$ and $x \in M$ such that $(R x+N: M) L \subseteq N$ and $L \nsubseteq N$. Then $\left(\frac{R x+N}{N}: \frac{M}{N}\right)\left(\frac{L}{N}\right)=N$. If $\frac{M}{N}$ is torsion free, then $\left(\frac{R x+N}{N}: \frac{M}{N}\right)=$ 0 . Hence $(R x+N: M)=0 \subseteq(N: M)$. If $\frac{M}{N}$ is compressible, there exists a monomorphism $f: \frac{M}{N} \rightarrow \frac{L}{N}$. Now $(R x+N: M) f\left(\frac{M}{N}\right) \subseteq N$ implies that $(R x+N: M) M \subseteq N$. Thus $(R x+N: M) \subseteq(N: M)$.

Corollary 2.12. Let $R$ be an integral domain and $M$ a torsion free $R$-module.

(1) Every direct summand of $M$ is *-primary.

(2) For every maximal ideal $m$ of $R$, the $R$-module $M_{m}$ is *-primary as an $R_{m}$-module. 


\section{3. *-PRIMARY SUBMODULES IN SOME FINITELY GENERATED MODULES}

In this section we characterize $*$-primary submodules in cyclic modules. Also we investigate $*$-primary submodules in the free $R$-module $R \oplus R$.

Proposition 3.1. Let $M$ be a cyclic $R$-module and $N$ a submodule of $M$. The following are equivalent:

(1) $N$ is a *-primary submodule and $\frac{R}{(N: M)}$ is a reduced ring.

(2) $N$ is a -primary submodule and $(N: M)$ is a semiprime ideal of $R$.

(3) $N$ is a strongly prime submodule of $M$.

Proof. $(3 \Rightarrow 1)$ Let $M=R x$ and let $N$ be a strongly prime submodule of $M$. Let $a+(N: M) \neq(N: M)$ and $n \in \mathbb{N}$ the smallest natural number such that $(a+(N: M))^{n} \subseteq(N: M)$. Then there exists $r_{0} \in R$ such that $a r_{0} x \notin N$. We have $\left(R a^{n-1} x+N: M\right) a r_{0} x \subseteq N$. Since $N$ is strongly prime, $a^{n-1} x \in N$. So $\left\langle a^{n-1} x\right\rangle \subseteq N$ and this is a contradiction.

$(1 \Rightarrow 2)$ and $(2 \Rightarrow 3)$ are clear.

Theorem 3.2. Let $M$ be a cyclic $R$-module and $N$ a proper submodule of $M$. The following are equivalent:

(1) $N$ is a primary submodule.

(2) $N$ is a *-primary submodule.

(3) $(N: M)$ is a primary ideal.

Proof. $(2 \Rightarrow 3)$ Let $M=R x, a b \in(N: M), a M \nsubseteq N$ and $b^{n} M \nsubseteq N$ for every $n \in \mathbb{N}$. Then there exist $r_{0}, r_{1} \in R$ such that $a r_{0} x \notin N, b^{n} r_{1} x \notin N$. We have $(R b m+N: M) a r_{0} x \subseteq N$ for every $m \in M$. Since $N$ is a $*$-primary submodule and $a r_{0} x \notin N,(R b m+N: M)^{k} \subseteq(N: M)$ for some $k \in \mathbb{N}$. So $(R b x+N: M)^{k} b x \subseteq N$. Therefore $b^{k} b x \in N$ and this is a contradiction.

$(3 \Rightarrow 2)$ Let $(R y+N: M) z \subseteq N$ and $z \notin N$. Then there exist $r, s \in R$ such that $(R r x+N: R x) s x \subseteq N$. Hence $(R r x+N: R x) s \subseteq(N: x)=(N: R x)=(N: M)$. Since $(N: M)$ is primary, $(R r x+N: R x) \subseteq \sqrt{(N: M)}$ and $r^{k} \in(N: M)$ for some $k \in \mathbb{N}$. Let $t_{1} t_{2} \cdots t_{k} \in(R r x+N: R x)^{k}$. Then $t_{1} t_{2} \cdots t_{k-1} t_{k} R x \subseteq$ $t_{1} t_{2} \cdots t_{k-1}(R r x+N) \subseteq r t_{1} t_{2} \cdots t_{k-1}(R x+N) \subseteq r^{2} t_{1} t_{2} \cdots t_{k-2}(R r x+N) \subseteq \cdots \subseteq$ $r^{k} R r x+N \subseteq N$.

( $3 \Leftrightarrow 1$ ) Let $N$ be a submodule of $M=R m$. Then for every $n \in N, n=r m$, we have $r \in(N: M)$ and hence $N=(N: M) M$, which implies that every cyclic module is multiplication, and we are done.

Corollary 3.3. Let $M$ be a cyclic $R$-module. Then $M$ is $*$-primary if and only if $\operatorname{ann}(M)$ is a primary ideal of $R$.

Fact 3.4. Let $M$ be a finitely generated $\mathbb{Z}$-module. Then $M \cong \mathbb{Z}_{p_{1}^{\alpha_{1}}} \oplus \mathbb{Z}_{p_{2}^{\alpha_{2}}} \oplus \cdots \oplus$ $\mathbb{Z}_{p_{k}^{\alpha_{k}}} \oplus \underbrace{\mathbb{Z} \oplus \mathbb{Z} \oplus \cdots \oplus \mathbb{Z}}_{n \text { times }}$, where $p_{1}, \ldots, p_{k}$ are prime numbers and $\alpha_{1}, \alpha_{2}, \ldots, \alpha_{k}$ are positive integers. Obviously $\underbrace{\mathbb{Z} \oplus \mathbb{Z} \oplus \cdots \oplus \mathbb{Z}}_{n \text { times }}$ is $*$-primary and $\mathbb{Z}_{p_{i}} \alpha_{i}$ are fully *-primary for each $i$. 
By means of the following proposition, which has an essential role in the remainder of this section, we can determine some $*$-primary submodules of a finitely generated free $R$-module $F$. We give its proof for the sake of completeness.

Proposition 3.5 ([10, Proposition 2.3]). Let $R$ be a domain and let $\left\{a_{i}\right\}_{i=1}^{n} \subseteq R$ be such that $R=R a_{1}+R a_{2}+\cdots+R a_{n}$. Then $R\left(a_{1}, \ldots, a_{n}\right)$ is a direct summand of the free $R$-module $F=R^{n}$.

Proof. $R=R a_{1}+R a_{2}+\cdots+R a_{n}$. Then there exist $s_{1}, s_{2}, \ldots, s_{n} \in R$ such that $1=s_{1} a_{1}+s_{2} a_{2}+\cdots+s_{n} a_{n}$. Let $N=\left\{\left(x_{1}, x_{2}, \ldots, x_{n}\right) \in F \mid s_{1} x_{1}+s_{2} x_{2}+\cdots+\right.$ $\left.s_{n} x_{n}=0\right\}$. Consider the functions $f: R \rightarrow R^{n}$ defined by $f(r)=r\left(a_{1}, \ldots, a_{n}\right)$ and $g: R^{n} \rightarrow R$ defined by $g\left(r_{1}, \ldots, r_{n}\right)=s_{1} r_{1}+s_{2} r_{2}+\cdots+s_{n} r_{n}$. The homomorphism $(g \circ f)$ is the identity. Then $R^{(n)}=\operatorname{Im} f \oplus \operatorname{ker} g=R\left(a_{1}, \ldots, a_{n}\right) \oplus N$.

In the following, we will study $*$-primary submodules in some finitely generated free modules; we get the same results obtained by Pusat-Yilmaz for prime submodules in [10].

Proposition 3.6. Let $R$ be a commutative ring and $F=R^{(n)}$ a finitely generated free $R$-module. The following statements hold:

(1) If $R$ is a domain and $\left\{a_{i}\right\}_{i=1}^{n} \subseteq R$ such that $R=R a_{1}+R a_{2}+\cdots+R a_{n}$, then $R\left(a_{1}, \ldots, a_{n}\right)$ is a *-primary submodule of $F$.

(2) Let $\left\{c_{1}, c_{2}, \ldots, c_{n}\right\} \subseteq F$ and $A=\left(\begin{array}{c}c_{1} \\ c_{2} \\ \vdots \\ c_{n}\end{array}\right)$. If $R \operatorname{det}(A)$ is a maximal ideal of $R$, then $N=R c_{1}+R c_{2}+\cdots+R c_{n}$ is a *-primary submodule of $F$.

Proof. (1) By Proposition 3.5, we have that $\frac{F}{R\left(a_{1}, \ldots, a_{n}\right)} \cong N$ is a torsion free module; then by Theorem 2.11 the submodule $R\left(a_{1}, \ldots, a_{n}\right)$ is $*$-primary.

(2) By [10, Proposition 3.7] $R \operatorname{det}(A) \subseteq(N: F) \subseteq \sqrt{R \operatorname{det}(A)}$. Since $R \operatorname{det}(A)$ is a maximal ideal of $R,(N: M)$ is too, and therefore $N$ is $*$-primary.

Proposition 3.7. Let $R$ be a commutative ring and let $a_{i}, b_{i} \in R(i=1,2)$ be such that $R=R b_{1}+R b_{2}$. Then $R\left(a_{1}, a_{2}\right)+R\left(b_{1}, b_{2}\right)$ is a *-primary submodule of $F=R \oplus R$ if and only if $R\left(a_{1} b_{2}-a_{2} b_{1}\right)$ is a primary ideal of $R$.

Proof. There exist elements $s_{1}, s_{2} \in R$ such that $1=s_{1} b_{1}+s_{2} b_{2}$. Then by Proposition 3.5. $F=L \oplus L^{\prime}$, where $L=R\left(b_{1}, b_{2}\right)$ and $L^{\prime}=\left\{(x, y) \in F \mid s_{1} x+s_{2} y=0\right\}$. We have $R\left(-s_{2}, s_{1}\right) \subseteq L^{\prime}$. Now $F=L+R\left(-s_{2}, s_{1}\right)$. It follows that $L^{\prime}=$ $\left(L \cap L^{\prime}\right)+R\left(-s_{2}, s_{1}\right)=R\left(-s_{2}, s_{1}\right)$. Now $F=L \oplus L^{\prime}$ and $N=L \oplus\left(N \cap L^{\prime}\right)$ give that $\frac{F}{N} \cong \frac{L^{\prime}}{N \cap L^{\prime}}=\frac{R\left(-s_{2}, s_{1}\right)}{R d\left(-s_{2}, s_{1}\right)} \cong \frac{R}{R d}$. Thus by Proposition 2.4. $N$ is a $*$-primary submodule of $F$ if and only if $R d$ is a primary ideal of $R$. 
In the above proposition the condition that $\left\{b_{1}, b_{2}\right\}$ is a spanning set of $R$ is a necessary condition. For, set $F=\mathbb{Z} \oplus \mathbb{Z}, N=\mathbb{Z}(6,6)+\mathbb{Z}(15,15)$; then $(\mathbb{Z}(1,2)+N$ : $\mathbb{Z} \oplus \mathbb{Z})(1,1) \subseteq N,(1,1) \notin N$ and $3^{n} \neq 0$ for every $n \in \mathbb{N}$.

Corollary 3.8. In the $\mathbb{Z}$-module $\mathbb{Z} \oplus \mathbb{Z}$, the submodule $\mathbb{Z}\left(a_{1}, a_{2}\right)$ such that $a_{1}, a_{2}$ are coprime is a $*$-primary submodule.

The converse of Proposition 3.6 (2) is generally not true. For consider $F=\mathbb{Z} \oplus \mathbb{Z}$ and $N=\mathbb{Z}(2,6)+\mathbb{Z}(1,3)$. By Proposition 3.7 the submodule $N$ of $F$ is a $*$-primary submodule but the zero ideal is not maximal.

Theorem 3.9. Let $R$ be a Noetherian domain and $R(\operatorname{det} A)$ a nonzero primary ideal of $R$, where $A=\left(\begin{array}{ll}a_{1} & a_{2} \\ b_{1} & b_{2}\end{array}\right)$. Then $N=R\left(a_{1}, a_{2}\right)+R\left(b_{1}, b_{2}\right)$ is a*-primary submodule of $R \oplus R$.

Proof. Let $\left(R\left(x_{1}, x_{2}\right)+N: R \oplus R\right)\left(y_{1}, y_{2}\right) \subseteq N$ and $\left(R\left(x_{1}, x_{2}\right)+N: R \oplus R\right)^{n} \nsubseteq$ $(N: M)$ for every $n \in \mathbb{N}$. Since $R$ is a Noetherian ring, there exists $r \in$ $\left(R\left(x_{1}, x_{2}\right)+N: R \oplus R\right)$ such that $r^{k} \notin(N: M)$ for every $k \in \mathbb{N}$ and $r\left(y_{1}, y_{2}\right) \in N$. Then $r\left(y_{1}, y_{2}\right)=s_{1}\left(a_{1}, a_{2}\right)+s_{2}\left(b_{1}, b_{2}\right)$ for some elements $s_{i} \in R(i=1,2)$. In matrix notation, we have $r\left(y_{1}, y_{2}\right)=\left(s_{1}, s_{2}\right)\left(\begin{array}{ll}a_{1} & a_{2} \\ b_{1} & b_{2}\end{array}\right)=\left(s_{1}, s_{2}\right) A$. Then $r\left(y_{1}, y_{2}\right) \operatorname{adj} A=\left(s_{1}, s_{2}\right) A \operatorname{adj} A=\left(s_{1}, s_{2}\right) \operatorname{det} A$. Let $\operatorname{adj} A=\left(\begin{array}{ll}b_{11} & b_{12} \\ b_{21} & b_{22}\end{array}\right)$. Then $r\left(y_{1}, y_{2}\right)\left(\begin{array}{ll}b_{11} & b_{12} \\ b_{21} & b_{22}\end{array}\right)=\left(s_{1}, s_{2}\right) D$, where $D=\operatorname{det} A$. So $r\left(y_{1} b_{1 j}+y_{2} b_{2 j}\right)=D s_{j} \in$ $\langle D\rangle$ for $j=1,2$. Since $\langle D\rangle$ is a primary ideal of $R,\left(y_{1}, y_{2}\right) B=D\left(t_{1}, t_{2}\right)$. Therefore $\left(y_{1}, y_{2}\right)=t_{1}\left(a_{1}, a_{2}\right)+t_{2}\left(b_{1}, b_{2}\right) \in N$.

\section{ACKNOWLEDGEMENTS}

The authors owe a great debt to the referee for having carefully read an earlier version of this paper and made significant suggestions for improvement.

\section{REFERENCES}

[1] M. Baziar and M. Behboodi, Classical primary submodules and decomposition theory of modules, J. Algebra Appl. 8 (2009), no. 3, 351-362. MR 2535994

[2] M. Behboodi, R. Jahani-Nezhad and M. H. Naderi, Classical quasi-primary submodules, Bull. Iranian Math. Soc. 37 (2011), no. 4, 51-71. MR 2915450

[3] J. Dauns, Prime modules, J. Reine Angew. Math. 298 (1978), 156-181. MR 0498715

[4] C.-P. Lu, Prime submodules of modules, Comment. Math. Univ. St. Paul. 33 (1984), no. 1, 61-69. MR 0741378.

[5] R. L. McCasland and P. F. Smith, Prime submodules of Noetherian modules, Rocky Mountain J. Math. 23 (1993), no. 3, 1041-1062. MR 1245463.

[6] R. L. McCasland and M. E. Moore, Prime submodules, Comm. Algebra 20 (1992), no. 6, 1803-1817. MR 1162609.

[7] M. E. Moore and S. J. Smith, Prime and radical submodules of modules over commutative rings, Comm. Algebra 30 (2002), no. 10, 5037-5064. MR 1940479

[8] A. R. Naghipour, Strongly prime submodules, Comm. Algebra 37 (2009), no. 7, 2193-2199. MR 2536911 
[9] A. R. Naghipour, Some results on strongly prime submodules, J. Algebr. Syst. 1 (2014), no. 2, 79-89. https://doi.org/10.22044/jas.2014.228

[10] D. Pusat-Yllmaz, On prime submodules of finitely generated free modules, Turkish J. Math. 27 (2003), no. 2, 329-342. MR 1986917

[11] P. F. Smith, Primary modules over commutative rings, Glasg. Math. J. 43 (2001), no. 1, 103-111. MR 1825725

[12] G. Ulucak and R. N. Uregen, A note on primary and weakly primary submodules, Eur. J. Pure Appl. Math. 9 (2016), no. 1, 48-56. MR 3462801.

Afroozeh Jafari

Department of Mathematics, Yasouj University, Yasouj, 75914, Iran

afroozehjafari@gmail.com

Mohammad Baziar ${ }^{\otimes}$

Department of Mathematics, Yasouj University, Yasouj, 75914, Iran

mbaziar@yu.ac.ir

Saeed Safaeeyan

Department of Mathematics, Yasouj University, Yasouj, 75914, Iran

safaeeyan@yu.ac.ir

Received: October 14, 2019

Accepted: June 30, 2020 